\title{
Atuação do enfermeiro na prevenção de doença renal crônica em portadores de diabetes: revisão integrativa
}

\author{
Nursing's activity in the prevention of chronic renal disease in diabetes carriers: integration \\ review
}

\section{Actuación del enfermero en la prevención de enfermedad renal crónica en portadores de diabetes: revisión integrative}

Francisca Jéssica de Sousa Oliveira ${ }^{1 *}$, Bruna Furtado Sena de Queiroz ${ }^{2}$, Kátia Lima Braga ${ }^{3}$, Paulo Ricardo Dias de Sousa ${ }^{1}$, lara Rege Lima Sousa ${ }^{4}$, Annielson de Souza Costa ${ }^{5}$, Marielle Cipriano de Moura $^{6}$, Bernarda de Oliveira Ramos Lima ${ }^{7}$, Ana Camila Pessoa Macário de Sousa ${ }^{1}$, Maria Aurislane da Silva Alves'.

\section{RESUMO}

Objetivo: Relacionar a atuação do enfermeiro na prevenção de doença renal crônica em portadores de diabetes. Métodos: Revisão bibliográfica realizada através da Biblioteca Virtual em Saúde (BVS), na Literatura Latino-Americana e do Caribe em Ciências da Saúde (LILACS), MEDLINE, Scientific Eletronic Library Online (SCIELO) e na Base de Dados de Enfermagem (BDENF).Resultados e Discussão: foram encontrados 77 artigos, dos quais apenas 8 foram usados para o estudo, foi criado um quadro onde foi organizado os artigos para facilitar o entendimento, $O$ papel da enfermagem na assistência ao paciente portador de DM e IRC é essencial, onde o profissional utiliza a sistematização da assistência de enfermagem garantindo ao cliente um cuidado integral e individualizado. Conclusão: O Enfermeiro desempenha um importante papel na sensibilização desses pacientes, pois pode ajudar na promoção da saúde ou na prevenção das doenças e na melhora da qualidade de vida dos indivíduos portadores de diabetes mellitus e doença renal crônica. É necessário capacitações, ofertados pelos governantes ou gestores de saúde aprimorando dessa formar os profissionais de saúde.

Palavras-chave: Doença renal, Prevenção, Enfermeiro.

\begin{abstract}
Objective: To relate the performance of the nurse in the prevention of chronic kidney disease in patients with diabetes. Methods: A bibliographical review was performed through the Virtual Health Library (VHL), the Latin American and Caribbean Literature in Health Sciences (LILACS), MEDLINE, the Scientific Electronic Library Online (SCIELO) and the Nursing Data base (BDENF). Results and Discussion: 77 articles were found, of which only 8 were used for the study, a table was created where articles were organized to facilitate understanding. The role ofnursing in the care of patients with DM and CRF is essential, where the professional uses the systematization of nursing care guarantee in the client integral and individualized care. Conclusion: The nurse plays na important role in the sensitization of these patients, since it can help in the promotion of healthor in the prevention of diseases and in the improvement of the quality of life of individuals with diabetes mellitus and chronic kidney disease. Or health managers improving this training of health professionals.
\end{abstract}

Keywords: Renal disease, Prevention, Nurse.

${ }^{1}$ Centro universitário Santo Agostinho (UNIFSA), Teresina- Piauí, *E-mail: fjessica_pi100@hotmail.com

${ }^{2}$ Faculdade Integral Diferencial - FACID-WYDEN, Teresina- Piauí.

${ }^{3}$ Ensino Superior pelo o Instituto de Ensino Superior Múltiplo- IESM

${ }^{4}$ Centro Universitário UINOVAFAPI (UNINOVAFAPI), Teresina- Piauí.

${ }^{5}$ Faculdade de Medicina da Universidade de São Paulo - FMUSP, São Paulo - SP.

${ }^{6}$ Universidade Estadual do Maranhão - UEMA, Timon- Maranhão.

${ }^{7}$ Associação de Ensino Superior do Piauí - AESPI, Teresina - Piauí. 


\section{RESUMEN}

Objetivo: Relacionar la actuación del enfermero en la prevención de enfermedad renal crónica en portadores de diabetes. Métodos: La revisión bibliográfica realizada a través de la Biblioteca Virtual en Salud (BVS), en la Literatura Latinoamericana y del Caribe en Ciencias de la Salud (LILACS), MEDLINE, ScientificEletronic Library Online (SCIELO) y en la Base de Datos de Enfermería (BDENF).Resultado y Discusión:Se han encontrado 77 artículos, de los cuales sólo 8 se utilizaron para el estudio, se creó un cuadro donde se organizaron los artículos para facilitar el entendimiento, El papel de la enfermería en la asistencia al paciente portador de DM e IRC es esencial, donde el profesional utiliza la atención sistematización de la asistencia de enfermería garantizando al cliente un cuidado integral e individualizado. Conclusión: El enfermero desempeña un importante papel en la sensibilización de estos pacientes, pues puede ayudar en la promoción de la salud o en la prevención de las enfermedades y en la mejora de la calidad de vida de los individuos portadores de diabetes mellitus y enfermedad renal crónica. Es necesario capacitaciones, ofrecidos por los gobernantes o gestores de salud mejorando de esa formación a los profesionales de salud.

Palabras clave: Enfermedad renal, prevención, Enfermera.

\section{INTRODUÇÃO}

A patologia renal consiste em lesão, podendo evoluir para perda progressiva e irreversível da função dos rins, no Brasil, a incidência e a prevalência de falência de função renal estão aumentando, constituindo atualmente um importante problema de saúde pública sendo ainda uma condição silenciosa, pois o paciente só começa a perceber que apresenta alguma alteração renal quando inicia o aparecimento dos sintomas urêmicos, ou seja, quando os rins perdem aproximadamente $50 \%$ de sua função (LEHMKUHL AM, et al., 2009).

Os rins são órgãos fundamentais para a manutenção da homeostase do corpo humano, sua função renal é avaliada pela filtração glomerular (TFG) e sua diminuição é observada na doença renal crônica (DRC) associada à perda das funções regulatória, excretória e endócrina dos rins. Com a redução progressiva da taxa de TFG observada na DRC e a consequente perda das funções regulatória, excretória e endócrina ocorre o comprometimento de todos os outros órgãos do corpo humano (BASTOS M, et al., 2004).

Alguns indivíduos apresentam suscetibilidade aumentada para DRC e são considerados grupos de risco. São eles: hipertensos, diabéticos, idosos, problemas cardiovascular, proteinúria, histórico familiar de doenças endêmicas e indivíduos com uso de nefrotóxicos. A doença renal diabética abrange os fatores hemodinâmicos, concentração plasmática dos produtos finais de glicolisação avançada, disfunção endotelial e entre outros (SESSO R, et al., 2008).

O diagnóstico da DRC baseia-se na presença de alterações do sedimento urinário (microalbuminúria, proteinúria, hematúria e leucocitúria) e redução da filtração glomerular avaliado pelo clearence de creatinina. A DRC é definida pela lesão do parênquima renal e/ ou pela diminuição funcional renal presentes por um período igual ou superior a três meses. Esta definição e classificação por estágio baseia-se na taxa de filtração glomerular (TFG) (BORTOLLO LA, 2008).

Para uma integralidade da assistência fazem se necessárias atividades de educação em saúde, uma vez que elas proporcionam a difusão dos conhecimentos entre profissionais da saúde e usuários. Sendo assim, por meio da educação em saúde os usuários constroem a autonomia e a emancipação para o autocuidado (MACHADO MD, et al., 2007). O objetivo da pesquisa é relacionar a atuação do enfermeiro na prevenção de doença renal crônica em portadores de diabetes.

\section{METODOS}

O presente estudo se caracteriza como uma revisão integrativa, no qual foram utilizadas as bases de dados eletrônicas, descritivas coletadas a partir das bases de dados: Literatura Latino-Americana e do Caribe em Ciências da Saúde (LILACS), (MEDLINE) e Base de Dados de Enfermagem (BDENF), utilizando-se conjuntamente os descritores em saúde: Doença renal; Prevenção; Enfermeiro. 
Os artigos foram triados pelos descritores citados, e a partir de um número máximo de artigos, os seguintes critérios foram sendo aplicados para refinar a pesquisa: textos completos disponíveis na integra. Foram incluídos estudos de caso, artigos completos disponíveis na integra, publicados no período de 2002 a 2019, no idioma português e inglês. Os critérios de exclusão foram: revisão de literatura, revisão sistemática, teses e dissertações, relatos de experiência, artigos cujo texto completo não fosse disponível na íntegra nem mesmo após busca em biblioteca de referência, ou seja, aqueles cuja aquisição, por algum problema técnico não fosse possível.

\section{RESULTADOS E DISCUSSÃO}

Foram localizados na Biblioteca Virtual em Saúde de enfermagem77artigos, sendo 63 (MEDLINE), 6 (LILACS) 5 na Base de Dados de Enfermagem (BDENF) e 3 no Scientific Eletronic Library Online (SCIELO). Logo após foram selecionados 08 adotados, a saber: artigos escritos nos idiomas português e inglês, recorte temporal de 2008 a 2019, para posteriormente serem mencionados nos resultados (Quadro 1).

De forma analítica segundo Fernandes LP, et al. (2018), o mesmo destaca que faz se necessária uma reorientação da prática educacional neste serviço, com o objetivo de ofertar melhor esclarecimentos sobre a DRC, e consequentemente, uma melhor qualidade de vida a estas pessoas. A uma necessidade de uma reorganização do planejamento de trabalho e definição das competências profissionais, visando as intervenções educacionais que favoreçam o autoconhecimento, a aceitação, a mudança de comportamento e o autocuidado da pessoa com DRC.

Respalda Stumm EM, et al. (2013), que a intervenção educacional, pode se constituir em: informações impressas com material educativo ou orientações personalizadas, individuais ou em grupos, com vistas a instrumentalizar o indivíduo para o autocuidado, como sujeito de sua terapêutica. No estudo de Suellen 2016 aborda que a equipe de saúde da atenção básica tem potencial para atuar na prevenção da DRC. É essencial adotar estratégias para equipar essas equipes para intervenções preventivas da DRC.

Segundo Mascarenhas NB, et al. (2011), os problemas identificados e na formulação dos diagnósticos apropriados à situação da paciente, fez-se necessário planejar uma assistência de enfermagem adequada à sua individualidade, de modo a garantir que não somente a situação clínica seja superestimada, mas que os aspectos biopsicossociais assumam posição privilegiada nas intervenções de enfermagem implementadas.

Para Costa EM e Carbone MH (2004), a equipe multiprofissional de uma Unidade de Saúde da Família deve trabalhar em conjunto para identificar os problemas da população, para que assim planeje e realize métodos que visem enfrentar os determinantes do processo saúde/doença, juntamente com a participação da comunidade. O papel da enfermagem na assistência ao paciente portador de DM e IRC é essencial, principalmente através de um cuidado de enfermagem sistematizado e coerente, com enfoque no ser humano e na sua família, de modo a tornar menos estressante e doloroso o tratamento desta complicação secundária a este distúrbio plurimetabólico denominado diabetes mellitus (MASCARENHAS NB, et al., 2011).

Nesse sentido Lima MA, et al. (2014), argumenta-se que as mudanças nos métodos assistenciais direcionadas a práticas educativas em saúde são ainda incipientes e têm pequena visibilidade no cenário nacional $O$ enfermeiro é um profissional essencial na educação em saúde e pode contribuir para a prevenção da DRC, empregando seus conhecimentos no atendimento aos pacientes diabéticos e hipertensos. (TRAVAGIM DS, et al., 2010). Corroborando com Mcclellan WM, et al. (2009), uma população de alto risco de 2 a 3 vezes mais chances para DRC consiste em familiares de primeiro ou segundo grau de pacientes em estágio final de doença renal terminal e faz-se necessário Programa de Educação da DRC nesta população.

Já no estudo de Burmeister JE, et al. (2011), observou quase 40\% de diabéticos entre os renais crônicos, os dados apresentaram um aumento impactante, sendo comparável ao encontrado em populações urbanas dos países desenvolvidos. No estudo de Barbosa ele observou uma análise que apontou para a adaptação, a negação, o ajuste social e a luta contra maus sentimentos que desvelaram a categoria $(R e)$ Construindo um novo estilo de vida: a adaptação. 
Quadro 1 - Artigos selecionados para leitura e análise.

\begin{tabular}{|c|c|c|c|}
\hline AUTOR & TÍTULO DO ARTIGO & OBJETIVO & METODOLOGIA \\
\hline $\begin{array}{l}\text { FERNANDES LP, } \\
\text { et al., } 2018\end{array}$ & $\begin{array}{l}\text { Necessidades de ações educativas } \\
\text { terapêuticas em um serviço de diálise } \\
\text { renal no Brasil. }\end{array}$ & $\begin{array}{l}\text { Investigar as dúvidas e/ou necessidades } \\
\text { das pessoas com DRC e a existência de } \\
\text { um programa educacional em um serviço } \\
\text { de HD. }\end{array}$ & $\begin{array}{l}\text { Participaram } 30 \text { voluntários com DRC em } \\
\text { tratamento HD, com a faixa etária variou entre } 40 \\
\text { a } 93 \text { anos. }\end{array}$ \\
\hline $\begin{array}{l}\text { SUELLEN AT, } \\
\text { et al., } 2016\end{array}$ & $\begin{array}{l}\text { Prevenção de doença renal crônica: } \\
\text { intervenção na prática assistencial } \\
\text { em equipe de saúde da família. }\end{array}$ & $\begin{array}{l}\text { Identificar os problemas prioritários em } \\
\text { relação à prevenção da Doença } \\
\text { Renal Crônica (DRC). }\end{array}$ & $\begin{array}{l}\text { Na coleta de dados, foram utilizadas } \\
\text { a observação, entrevista semiestruturada com } \\
11 \text { profissionais. }\end{array}$ \\
\hline MASCARENHAS NB, et al., 2011 & $\begin{array}{l}\text { Sistematização da assistência de } \\
\text { enfermagem ao portador de diabetes } \\
\text { mellitus e insuficiência renal crônica. }\end{array}$ & $\begin{array}{l}\text { Relatar a aplicação da Sistematização da } \\
\text { Assistência de Enfermagem na } \\
\text { assistência a um paciente portador de } \\
\text { DM e IRC. }\end{array}$ & $\begin{array}{l}\text { Uma participante com } 81 \text { anos, portadora de DM } \\
\text { e IRC foi acompanhada durante o período de } \\
\text { admissão até sua alta hospitalar. }\end{array}$ \\
\hline LIMA MA, et al., 2014 & $\begin{array}{l}\text { Educação em saúde para pacientes } \\
\text { em hemodiálise. }\end{array}$ & $\begin{array}{l}\text { Relatar o conhecimento dos pacientes } \\
\text { com insuficiência renal crônica. }\end{array}$ & $\begin{array}{l}\text { Estudo qualitativo, do tipo pesquisa-ação, } \\
\text { realizado em uma clínica de hemodiálise, no } \\
\text { interior do Piauí/PI, com } 08 \text { participantes. }\end{array}$ \\
\hline TRAVAGIM DS. et al., 2010 & $\begin{array}{l}\text { Prevenção e progressão da doença } \\
\text { renal crônica: atuação do enfermeiro } \\
\text { com diabéticos e hipertensos }\end{array}$ & $\begin{array}{l}\text { Objetivou-se descrever a atuação dos } \\
\text { enfermeiros na prevenção e na } \\
\text { progressão da DRC, da atenção básica à } \\
\text { saúde, com pacientes diabéticos e } \\
\text { hipertensos. }\end{array}$ & $\begin{array}{l}12 \text { enfermeiras atuantes nos núcleos de saúde } \\
\text { da família e nas UBS. }\end{array}$ \\
\hline $\begin{array}{l}\text { TRAVAGIM DS e KUSUMOTA L. } \\
2009\end{array}$ & $\begin{array}{l}\text { Atuação do enfermeiro na prevenção } \\
\text { e progressão da doença renal } \\
\text { crônica. }\end{array}$ & $\begin{array}{l}\text { Identificar os conhecimentos dos } \\
\text { enfermeiros atuantes em serviços de } \\
\text { atenção básica à saúde acerca da } \\
\text { prevenção e progressão da doença renal } \\
\text { crônica (DRC), }\end{array}$ & $\begin{array}{l}\text { Foi utilizada a abordagem metodológica } \\
\text { qualitativa, sendo o método de coleta de dados a } \\
\text { história oral temática. A coleta de dados foi } \\
\text { realizada com } 12 \text { enfermeiras atuantes nos } \\
\text { núcleos de saúde da família e UBS. }\end{array}$ \\
\hline $\begin{array}{l}\text { BURMEISTER JE, } \\
\quad \text { et al., } 2011\end{array}$ & $\begin{array}{l}\text { Prevalência de diabetes mellitus em } \\
\text { pacientes renais crônicos sob } \\
\text { hemodiálise em Porto Alegre, Brasil }\end{array}$ & $\begin{array}{l}\text { Estimar a prevalência de DM na } \\
\text { população adulta de pacientes em } \\
\text { tratamento hemodialítico por IRC }\end{array}$ & $\begin{array}{l}\text { Estudo transversal descritivo, quantitativo e } \\
\text { analítico, com inquérito sobre a prevalência de } \\
\text { DM entre os } 1.288 \text { pacientes em tratamento por } \\
\text { hemodiálise. }\end{array}$ \\
\hline $\begin{array}{c}\text { BARBOSA GS e VALADARES } \\
\text { GV. } 2009\end{array}$ & $\begin{array}{l}\text { Hemodiálise: estilo de vida e a } \\
\text { adaptação do paciente. }\end{array}$ & $\begin{array}{l}\text { Identificar como o paciente dependente } \\
\text { de hemodiálise enfrenta no dia-a-dia o } \\
\text { processo de doença, considerando } \\
\text { atitudes, comportamentos e práticas. }\end{array}$ & 10 participantes, dependentes de hemodiálise. \\
\hline
\end{tabular}

Fonte: Elaborada pelos autores, 2019.

REAS/EJCH | Vol.Sup.30 | e927 | DOI: https://doi.org/10.25248/reas.e927.2019 Página 4 de 5 
Os cuidados de enfermagem têm sete funções diferentes: ajudar; educar; diagnosticar, acompanhar e monitorar o doente; solucionar situações de evolução rápida; administrar e acompanhar protocolos terapêuticos; assegurar e acompanhar a qualidade dos cuidados de saúde e assegurar e acompanhar as competências no âmbito da organização dos serviços de saúde (ROSO CC, et al., 2013).

\section{CONCLUSÃO}

O enfermeiro desempenha um importante papel na sensibilização desses pacientes, atuando na promoção da saúde e/ou prevenção das doenças, refletindo na melhoria da qualidade de vida dos portadores de DM e renais crônicos. É necessário capacitações, ofertados pelos governantes ou gestores de saúde aprimorando dessa forma os profissionais de saúde. Novos estudos são necessários para se obter maiores conhecimentos da efetividade e estratégias de promoção em saúde que vise prevenir doenças endêmicas como a DM, evitando que a mesma evolua para uma insuficiência renal e entre outras patologias. Espera-se que esses resultados possam embasar planejamento de programas voltados para a educação em saúde e intervenção, a fim de prevenir ou melhorar as condições de saúde dos indivíduos portadores de diabetes e os nefrológicos.

\section{REFERÊNCIAS}

1. BASTOS M et al. Doença renal crônica: problemas e soluções. J Brasileira de Nefrologia, 2004; 26(4): $202-215$.

2. BARBOSA GS, VALADARES GV. Hemodiálise: estilo de vida e a adaptação do paciente. Acta paul. enfermagem, 2009; 22(1): 524-527.

3. BORTOLOTTO LA. Hipertensão arterial e insuficiência renal crônica. Revista brasileira de hipertensão, 2008; 15(3):152- 155.

4. BURMEISTER JE, et al. Prevalência de diabetes mellitus em pacientes renais crônicos sob hemodiálise em Porto Alegre. Jornal Brasileiro de Nefrologia, 2012; 34(2):117-121.

5. COSTA EMA, CARBONE MH. Saúde da Família: uma abordagem interdisciplinar. 2004.

6. FERNANDES LP, MARINS KYM, CARMO HO et al. Necessidades de ações educativas-terapêuticas em um serviço de diálise renal no Brasil. Enfermería Nefrológica, 2018; 21(1): 53-62.

7. LEVEY AS, CORESH J, BOLTON K et al. K/DOQI clinical practice guidelines for chronic kidney disease: evaluation, classification, and stratification. American Journal of Kidney Diseases, 2002; 39(2).

8. LEHMKUHL AM, ALFREDO JM, MACHADO MO. Estudo da prevalência de óbitos de pacientes com doença renal crônica associada à doença mineral óssea. Revista Brasileira de Nefrologia, 2009; 31(1): 10-7.

9. LIMA MA, SOUSA GR, SOUSA AM, et al. Educação em saúde para pacientes em hemodiálise. Journal of Nursing UFPE/Revista de Enfermagem, 2014; 8(6).

10. MACHADO MDF, MONTEIRO EM, QUEIROZ DT, et al. Integralidade, formação de saúde, educação em saúde e as propostas do SUS: Uma revisão conceitual. Ciência \& saúde coletiva, 2007; 12(2): 335-342.

11. MASCARENHAS NB, PEREIRA Á, SILVA RSD. Sistematização da assistência de enfermagem ao portador de diabetes mellitus e insuficiência renal crônica, 2011.

12. MCCLELLAN WM, SATKO SG, GLADSTONE E, et al. Individuals with a family histoty of ESRD are a high-risk population for CKD: implications for targeted surveillance and intervention. American Journal of Kidney Diseases, 2009; 53(1):100-106.

13. OLIVEIRA GKS, OLIVEIRA ER. Assistência de Enfermagem ao Portador de Diabetes Mellitus: Um Enfoque na Atenção Primária em Saúde. Revista Eletrônica de Ciências, 2010; 3(2).

14. ROSO CC, BEUTER M, KRUSE MHL et al. O cuidado de si de pessoas em tratamento conservador da insuficiência renal crônica. Texto \& contexto enfermagem, 2013; 22(3): 739-745.

15. SESSO R, LOPES AA, THOMÉ AS, et al. Relatório do Censo Brasileiro de Diálise, 2008 Relatório do Censo Brasileiro de Diálise. Revista Brasileira de Nefrologia, 2008; 30(4): 233-8.

16. SUELLEN AT, OLIVEIRA OA, SERRA GA, et al. Chronic kidney disease prevention: intervention in assistance practice in a Family health team. Journal of Nursing UFPE/Revista de Enfermagem UFPE, 2016; 10(9).

17. STUMM EMF, KIRCHNER RM, BELASCO A, et al. Validation of a manual for patients with hyperphosphatemia. J Nurs UFPE, 2013; 7(9): 5485-9.

18. TRAVAGIM DSA, KUSUMOTA L. Atuação do enfermeiro na prevenção e progressão da doença renal crônica. Revista Enfermagem UERJ, 2009; 17(3): 388-393.

19. TRAVAGIM DSA, KUSUMOTA L, TEIXEIRA CRD, et al. Prevenção e progressão da doença renal crônica: atuação do enfermeiro com diabéticos e hipertensos, 2010: 1-7. 